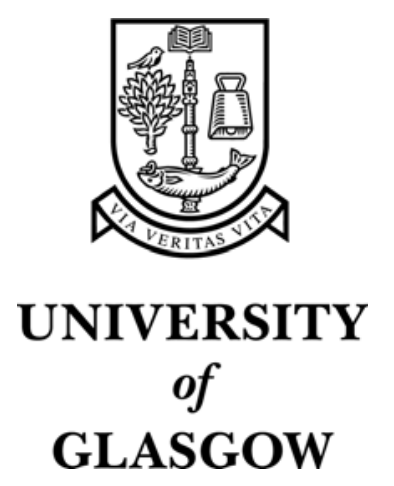

Yu, W. and Ramloll, R. and Brewster, S.A. and Riedel, B. (2001)

Exploring computer-generated line graphs through virtual touch. In, 6th International Symposium on Signal Processing and its Applications (ISSPA), 13-16 August 2001 Vol 1, pages pp. 72-75, Kuala Lumpur, Malaysia.

http://eprints.gla.ac.uk/3202/ 


\title{
EXPLORING COMPUTER-GENERATED LINE GRAPHS THROUGH VIRTUAL TOUCH
}

\author{
Wai Yu, Ramesh Ramloll, Stephen Brewster, Beate Ridel* \\ Department of Computing Science, "Department of Psychology \\ University of Glasgow, G12 8QQ, UK \\ rayu@dcs.gla.ac.uk, http://www.dcs.gla.ac.uk/ rayu/home.html
}

\begin{abstract}
This paper describes the development and evaluation of a haptic interface designed to provide access to line graphs for bind or visually impaired people. Computer-generated line graphs can be felt by users through the sense of touch produced by a PHANToM force feedback device. Experiments have been conducted to test the effectiveness of this interface with both sighted and blind people. The results show that sighted and blind people have achieved about $89.95 \%$ and $86.83 \%$ correct answers respectively in the experiment.
\end{abstract}

\section{INTRODUCTION}

We are currently developing a multimodal visualisation system for blind and visually impaired people. The main objective of this work is to provide blind people with access to graphs and tables by using virtual reality technologies. Haptics and sound are two main modalities used in our system. At present, the use of haptics and audio to present information to the users is being investigated separately. This is aiming at determining each modality's strengths and limitations so that they can be integrated effectively in the multimodal system. In this paper, we introduce our development in haptic line graphs with details of design and implementation issues. Moreover, results obtained from evaluations, which have been conducted on both sighted and blind people, are discussed.

Traditionally, graphs are presented to blind people on raised paper or other custom-made materials [4]. The production process can be very tedious and laborious, and the finished products often suffer from degradation after frequent use. Research has been done to computerise Braille and raised graphs so that they can be stored digitally and re-printed if necessary [5]. Successful use of tactile graphs depends on experience and competence of the user. Some tablets have been developed for exploring graphs or schematic diagrams $[1,7]$. They either use a matrix of actuated pins/cells or touch input and speech output to present graphical information. These kinds of device suffer from high cost, low resolution and inflexibility. As for audio-based tablets, each new diagram has to be programmed beforehand which is time consuming and it is difficult to change the diagram.
Since the advent of force feedback devices, virtual haptic feedback has become feasible and has potential in many applications. Besides simpler production process of virtual objects, storage and carrying of digital data are more convenient than conventional methods. Several attempts have been made to present 3D objects, scientific data and mathematical functions to blind people using haptic feedback $[3,6,9]$. However, the development of the haptic interface is the major concern rather than the usability issues. In order to address this problem, research work is needed to investigate the effectiveness of the new form of haptic feedback on presenting information to blind people. Studies have been done in the Department of Computing Science at the University of Glasgow $[2,10]$ to investigate the development of haptic graphs including line graphs and bar charts. The experimental results indicate that in order to make haptic graphs suitable for blind people, conventional tactile graph representation techniques cannot be adopted completely. The major difference is that in the haptic object modelling technique, objects are engraved into the surface rather than raised up. This is due to the limitation of current force feedback devices which provide a single point of contact. Concaved surface is more effective to keep the device pointer in contact. Other modifications are needed to compensate for the limited bandwidth possessed in a single point of contact in which only small amount of information can be conveyed at a time. Based on the experience obtained from these previous studies, a new set of haptic line graphs has been developed by using a modified haptic object modelling technique.

\section{HAPTIC LINE GRAPH DESIGN}

The haptic interface is built on a Windows NT system which consist of a PIII $550 \mathrm{MHz}$ PC and a SensAble PHANTOM 1.5 force feedback device. The computergenerated line graphs are constructed by using the GHOST SDK. Users feel the line graphs through the end-effector of the PHANTOM. The PHANTOM-controlled pointer moves in a three dimensional space in which all virtual objects are located on the background. The lines have a Vshape cross-section and are attached to the background. An illustration of a line graph model is given in Figure 1. Polygons are used to construct the channels and the inner surface is defined as touchable by the PHANToM so that 
the pointer can penetrate from the outside and become retained in the inside. By moving the pointer along the channel, users can trace the path of the line. In order to handle multiple lines on a graph, friction keys, which are the frictional property of the polygon surface, are used as a distinctive feature. Therefore, by judging the degrees of friction, users can distinguish different lines.

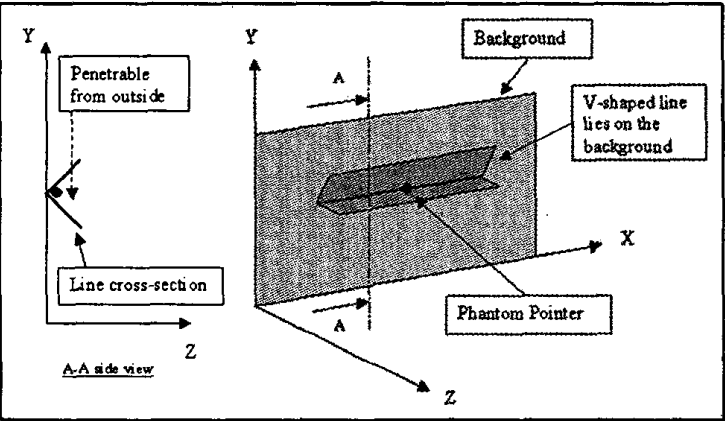

Figure 1. Haptic line graph model.

The reason for making a $\mathrm{V}$-shape channel is to retain the pointer on the line. In our previous studies, we found that users had problems with keeping the pointer on raised objects $[2,10]$. A'concave shape can effectively solve this problem. Instead of engraving lines into the surface, we decided to construct them on the surface due to several advantages. Firstly, we can take full advantage of GHOST SDK's support of polygons which have the force model defined. Secondly, addition and removal of lines to and from the graph are relatively simple and without the need to recreate the whole haptic scene. Finally, it provides the flexibility that simple straight lines or smooth curves can be constructed by putting the desired number of polygons together.

\section{PILOT STUDY}

\subsection{Experiment Design}

A pilot study has been conducted to validate the design of the haptic interface. 15 sighted subjects took part and they were Computing Science students at the University of Glasgow. Two sets of six graphs were designed for the experiment. Each graph in the first set contained up to three separated lines whilst those in the second set contained two intersected lines. Two sample graphs are given in Figure 2. Participants had four minutes to explore each graph. Within the time limit, they needed to perform a set of tasks which included:

- Counting the number of lines.

- Identifying the line smoothness.

- Counting the number of bends on the line.

- Counting the number of intersections, if any.

Moreover, they needed to remember the relative position of the lines and their location on the graph so that they could sketch the graphs after the exploration. This set of tasks was aimed at testing the accuracy of the information conveyed through the interface. Generally, line graphs are used to present data trends and provide difference comparisons. By asking about features and locations of the lines, the usefulness of the interface can be revealed. In addition, several measurements were taken in the experiment. They are including:

- Task completion time.

- A log file containing pointer positions.

- A questionnaire regarding the interface design.

- NASA Task Load Index test.

The NASA Task Load Index (TLX) [8] indicates the workload placed on participants in the experiment and it contains six categories: mental demand, physical demand, temporal demand, effort, performance and frustration. Three practice graphs were given to the participants before the experiment in order to familiarise them with the haptic interface and experiment procedure.

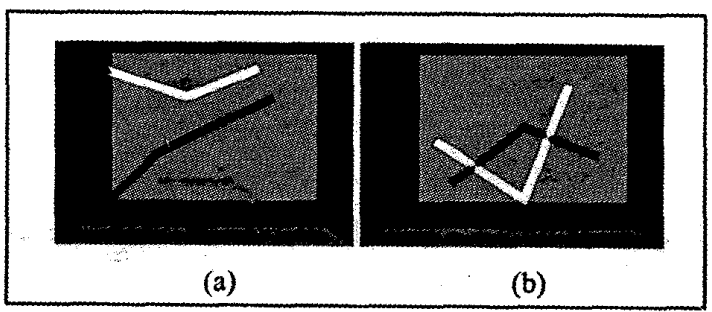

Figure 2. Experiment graphs. (a) Non-intersected graph, (b) Intersected graph.

\subsection{Results}

Table 1 . lists the summarized results. The figures show the percentage of correct answers to the questions asked in the task. The overall correct answers in the first and second set of graphs were $85.56 \%$ and $94.07 \%$ respectively. The difference between these two groups was significant $\left(T_{14}=3.97, p=0.0014\right)$. A high percentage of correct answers was obtained from the identification of frictional properties (Table 1). Overall results obtained in the first and second set of graphs are compared in terms of score/correct answers, task completion time and workload index (Figure 3). From the graph, the mean task completion time is similar in both sets whilst the workload is higher in the second set. The overall workload index is 10.6 and 12.21 in the first and second set respectively, and the difference is significant $\left(\mathrm{T}_{14}=3.75, \mathrm{p}=0.0022\right)$.

\begin{tabular}{|l|l|l|l|l|}
\hline \multirow{2}{*}{\multicolumn{2}{|c|}{ Questions }} & \multicolumn{2}{c|}{ Non-Intersected } & \multicolumn{2}{c|}{ Intersected } \\
\cline { 2 - 5 } & Mean & Std Dev & Mean & Std Dev \\
\hline No. of lines & 86.67 & 15.69 & 96.67 & 9.34 \\
\hline Friction keys & 94.44 & 6.03 & 98.89 & 4.3 \\
\hline No. of bends & 76.11 & 16.33 & 88.89 & 15 \\
\hline No. of intersections & N.A. & N.A. & 92.22 & 13.9 \\
\hline
\end{tabular}

Table 1. Sighted participants' correct answers (all figures in \%).

\subsection{Discussion}

The findings of the experiment show that participants' performance is better in the second set of graphs. The amount of practice they had after completing the first set of graphs was the main contribution. The significant difference in the workload index reveals that participants 
had to work harder in order to achieve better results. Among the six categories of workload index, mental demand and effort received highest ratings around $69.5 \%$ and $56.5 \%$. This may indicate sighted people are not used to perceiving information by touch alone. In summary, results obtained in the pilot study are promising as participants can use the haptic interface to get high percentage of correct answers.

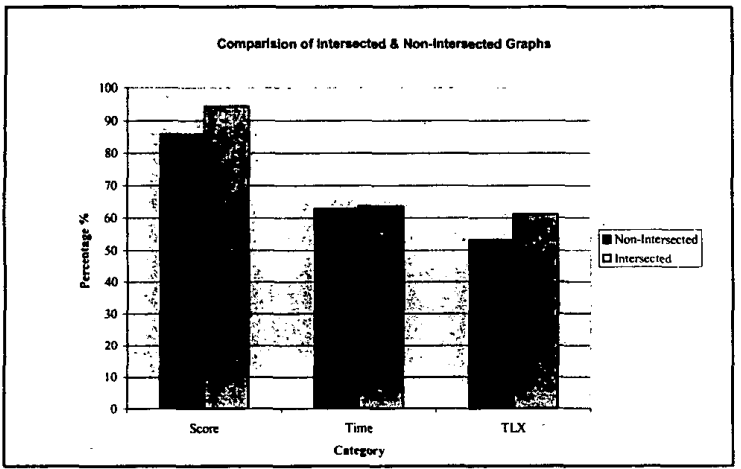

Figure 3. Overall results of sighted participants.

Besides analyzing the experiment data, several problems with the haptic line graph design were identified. Friction keys were regarded as an effective feature in the informal questionnaire. On the other hand, it could hinder participants' movement on a line, especially when the line had sharp bends and strong friction. This combination could mislead the participants into misjudging the sharp bend as the end of the line. This gave participants an incomplete image of the graph.

Perceiving information through touch is much slower than vision. The picture of the line graph is built up by pieces of information picked up through the finger tip over a period of time. Although the accuracy in counting the number of lines is high, $86.67 \% \& 96.67 \%$ for the first and second set respectively, it is very time consuming for the participants to determine whether a line has been explored before. In order to overcome this problem, additional cues are required to assist the user.

\section{EVALUATION}

\subsection{System Improvements}

Based on the results and observations taken in the pilot study, several additional features were planned to improve the haptic interface. However, only two of them were implemented in time for the evaluation experiment due to time constraints. They were the instant line identity and line end point indication. The lines were numbered and whenever the pointer touched a line, PHANToM generated a sequence of clicks (a gentle movement in the $\mathrm{z}$ axis). The number of clicks is same as the line number therefore users can determine which line it is. The line end point indication is a continuous vibration which only occurs when the pointer reaches the end of a line. It is designed to reduce the confusion between sharp bends and line end points.

\subsection{Experiment Setup}

The evaluation was conducted on 15 blind people. They were recruited from the Royal Blind College at Hereford and the Royal National Institute for the Blind at Peterborough. Some modifications were made to the experiment setup. The graphs used in the pilot study were used again but the four simplest graphs were taken out to shorten the length of the experiment. They were arranged in two groups and each had two intersected and two nonintersected graphs. The difference between two groups in this case is that one group had the enhanced features while the other one did not. The order of taking group one and two was -randomized for each participant so that the learning effect was minimized. Experiment procedure and measurements were similar to those in the pilot study except that participants were not asked to sketch the graph after explorations. Instead, a think aloud method was used to test the graph image perceived by the participants.

\subsection{Results}

The number of correct answers achieved by the participants is listed in Table 2. The amount of correct answers obtained in group one and two are very similar and they are $87.45 \%$ and $86.2 \%$ respectively. Again, participants did very well at identifying the frictional properties. Figure 4 shows the overall results in score, task completion time and workload index. The score obtained in group two is slightly lower than those in group one. It shows that enhanced graphs did not give participants significant advantages. Furthermore, task completion time and workload index are slightly lower in group two again and that means participants needed less time and work less harder in group two. However the difference between two sets of data in terms of three categories is not significant.

\begin{tabular}{|l|l|l|l|l|}
\hline \multirow{2}{*}{\multicolumn{1}{|c|}{ Questions }} & \multicolumn{2}{c|}{ Standard } & \multicolumn{2}{c|}{ Enhanced } \\
\cline { 2 - 5 } & Mean & Std Dev & Mean & Std Dev \\
\hline No. of lines & 86.67 & 28.14 & 88.33 & 16 \\
\hline Friction keys & 95.56 & 10.11 & 94.81 & 8.26 \\
\hline No. of bends & 79.26 & 28.44 & 73.33 & 30.23 \\
\hline No. of intersections & 88.33 & 18.58 & 88.33 & 16 \\
\hline
\end{tabular}

Table 2. Blind participants' correct answers (all figures in \%).

\subsection{Discussion}

The participants did not achieve the same level of performance on the enhanced graphs which means that newly implemented features had little effect in helping people to explore the graphs. However, the usefulness of the enhancement is reflected in other areas such as variance of correct answers, mental demand of the workload index and users' feedback from the questionnaire. A significance test on the variance of correct answers to number of lines produce $F_{14}=3.09$, $\mathrm{p}=0.021$ which indicates that variance 0.41 in the enhanced group is significantly lower than the variance 1.27 in the standard group. The line identity helps people who do not perform that well in the standard group to improve. Similarly to the pilot study, mental demand and effort receive highest overall ratings. In mental demand, 
International Symposium on Signal Processing and its Applications (ISSPA), Kuala Lumpur, Malaysia, 13 - 16 August, 2001. Organized by the Dept. of Microelectronics and Computer Engineering, UTM, Malaysia and Signal Processing Research Centre, QUT, Australia

participants rated $61.67 \%$ for group one and $51 \%$ for group two and the difference is statistically significant $\left(\mathrm{T}_{14}=2.35, \mathrm{p}=0.034\right)$. In the effort category, $57.33 \%$ and $53.67 \%$ were obtained for group one and two respectively. Therefore, although the effect of the enhancements on participants' performance is not so obvious, they have helped minimizing the variance between individuals and reduced mental workload.

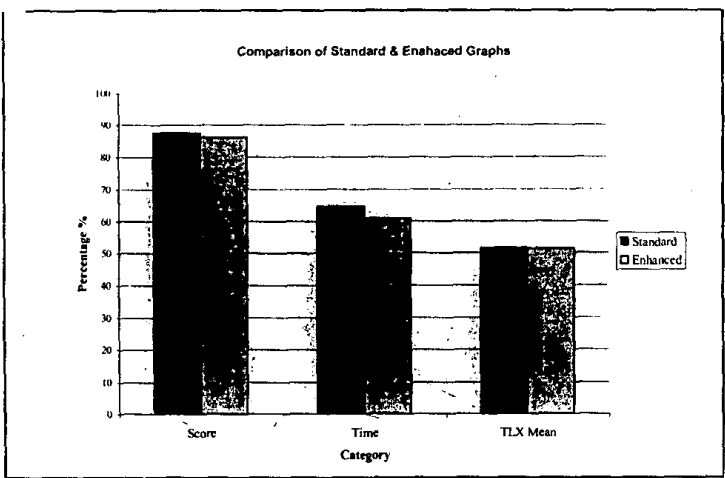

Figure 4. Overall results of blind participants.

Blind participants did not achieve the same level of score as the sighted people. This may due to the difference between sighted and blind people as well as the wider range of blind people recruited for the experiment. Sighted participants were all computing science students who are more familiar with graphs and capable of using the force feedback device. Whereas, the majority of the blind participants were studying a diploma course in the Royal Blind College and there is a big difference between their age, education background and familiarity to graphs. Although, blind participants achieved similar score, the task completion time is similar to sighted people and the workload is also lower.

We are currently integrating auditory feedback into our haptic interface so that problems that cannot be solved easily in haptics can be dealt with in another medium. From our experiments, haptic feedback is found to be more useful for guidance and assisting users' navigation on the graphs. It is not good at presenting exact data values to the user. Moreover, if we put in too much information through haptics, its narrow bandwidth can be easily overloaded. Therefore by using another sensory modality, some workload can be shifted across and each of them can do what they'are good at.

\section{CONCLUSION}

The usefulness of the haptic interface has been confirmed in both a pilot study and a full evaluation. Majority of blind participants can use the interface successfully and perceive correct information about the graphs. Friction keys are very effective for the users to distinguish different lines. Enhanced features can minimize the difference between individual's capabilities and reduce mental workload. Some problems exist in the current design of the haptic line graph such as the combinational effect of sharp corner and strong friction, path change at intersections. Further improvement is needed to solve these problems.

\section{ACKNOWLEDGEMENTS}

The authors would like to thank the Royal Blind College and the Royal National Institute for the Blind for their participations in the experiments. This research work is funded by EPSRC Grant GR/M44866, ONCE (Spain) and Virtual Presence Ltd.

\section{REFERENCES}

[1] Blenkhorn P. and Evans D.G., Using speech and touch to enable blind people to access schematic diagrams. Journal of Network and Computer Applications, 1998. 21: p. 17-29.

[2] Brewster S. A. and Pengelly H. Visual impairment, virtual reality and visualisation. In First international workshop on usability evaluation for virtual environments. 1998. p. 24-28, Leicester UK: British Computer Society.

[3] Colwell C., Petrie H., Kornbrot D. Hardwick A. and Furner S. Haptic virtual reality for blind computer users. In ACM Conference on Assistive Technologies. 1998. p. 92-99, Marina del Rey, CA, USA: ACM.

[4] Edmar, P.K., Tactile graphics. 1991: American Foundation for the Blind.

[5] Francois, G., Computerised Braille production: past, present, future. Computers for handicapped persons, 1989: p. 52-56.

[6] Grabowski N. A. and Barner K.E. Data visualisation methods for the blind using force feedback and sonification. In SPIE-Int. Soc. Opt. Eng. Proceedings of SPIE - the International Society for Optical Engineering. 1998. p. 131-139; Boston, MA, USA: SPIE.

[7] Lee S., Wiker S. F., Vanderheiden G. C. Interactivè haptic interface: two-dimensional form perception for blind access to computers. in 5th International conference on human computer interaction. 1993. p. 190-195.

[8] Hart S. G. and Wicken S. C., Workload assessment and predication, in MANPRINT, an approach to systems integration. 1990, Van Nostrand Reinhold: New York.

[9] Van Scoy F., Kawai T., Darrah M. and Rash C., Haptic display of mathmatical functions for teaching mathematics to students with vision disabilities: design and proof of concept. In First international workshop on haptic human-computer interaction. 2000. p. 78-81, Glasgow UK.

[10] Yu W., Ramloll R, and Brewster S. A. Haptic graphs for blind computer users. In First international workshop on haptic human-computer interaction. 2000. p. 102-107, Glasgow UK. 